\title{
Le DATATOP persiste et signe... un texte sensiblement différent!
}

Il y a trois ans (voir $\mathrm{m} / \mathrm{s} n^{\circ} 9$, vol. 5, p. 685), nous avions présenté les résultats d'une large étude multicentrique réalisée aux États-Unis chez des patients atteints de maladie de Parkinson à une phase très précoce, le DATATOP. Le but de cette étude était d'apprécier les effets d'un traitement de première intention par un inhibiteur de la monoamine oxydase B, le déprényl (sélégiline).

L'intérêt particulier apporté à cette drogue - qui justifiait l'inclusion d'un très grand nombre de patients (800) et la participation de la plupart des ténors de la neurologie du Parkinson outreAtlantique - découlait en grande partie de l'hypothèse totalement originale qui sous-tendait son utilisation. Les fondements de l'étude étaient, en effet, à trouver dans une très abondante littérature expérimentale consacrée à un agent toxique, le MPTP, capable de provoquer la mort des neurones dopaminergiques de la substance noire et, par là, un syndrome parkinsonien. L'étude des mécanismes d'action du toxique avait conduit à la démonstration que le MPTP n'était pas luimême neurotoxique mais qu'il était catabolisé in vivo en MPP +, un cation qui pouvait bloquer la chaîne respiratoire mitochondriale [1]. Or, la chaîne qui conduit du MPTP au MPP + passe par la monoamine oxydase B. Quoique l'existence d'un agent toxique endogène dans la maladie de Parkinson idiopathique reste à démontrer, le blocage de l'enzyme par un inhibiteur sélectif (sans action sur la monoamine oxydase A) et irréversible, paraissait donc très intéressant à tester cliniquement. Ce blocage pouvait, en effet, créer des conditions métaboliques telles que des agents toxiques ne puissent être formés et, donc, procurer une thérapeutique préventive de la neurodégénérescence.

Les premiers résultats, dont nous nous étions faits l'écho, démontraient que la prise quotidienne de $10 \mathrm{mg}$ de déprényl permettait effectivement de $\mathrm{m} / \mathrm{s} n^{\circ} 3$ vol. 9, mars 93 retarder l'évolution de la maladie et la première prise de L-Dopa dont la date était le end-point de l'étude pour chaque malade. Suivant les conclusions des auteurs, ce résultat pouvait être interprété comme la conséquence d'un retard de la mort neuronale lié à la neuroprotection créée par le déprényl. On aurait pu, de façon alternative, suggérer que le déprényl avait un effet symptomatique antiparkinsonien propre - en plus ou à la place des effets neuroprotecteurs -, mais une étude pilote réalisée par l'un des participants du DATATOP (et, depuis, quelques autres études limitées) semblait l'avoir exclu [2, 3].

La publication - avant terme - des résultats obtenus était, il y a trois ans, une première conséquence de l'action d'un comité indépendant de surveillance de l'étude multicentrique. Une seconde conséquence de cette action a été un changement des protocoles, dont on voit aujourd'hui paraître les effets [4]. Le comité d'experts indépendants ne s'était pas totalement satisfait des résultats de l'étude pilote prouvant l'inefficacité symptomatique du déprényl. Le DATATOP ayant une cohorte suffisamment importante pour déceler un effet faible mais statistiquement significatif, le comité a demandé la réalisation d'un test capable de différencier effets symptomatique et neuroprotecteur : une fenêtre thérapeutique de deux mois. Le fondement de ce changement de protocole était que les constantes de temps des deux effets seraient vraisemblablement différentes et qu'un laps de temps assez court permettrait d'isoler une action "aiguë " du produit, probablement symptomatique, d'une action "chronique" de neuroprotection. Cette fenêtre s'ouvre aujourd'hui sur une rectification majeure de l'interprétation des premiers résultats publiés... et des fondements mêmes de l'étude. Car le déprényl a clairement une action symptomatique antiparkinsonienne : la mise en cuvre du traitement induit une amélioration notable des signes cliniques; l'interruption du traitement provoque en quelques semaines une aggravation significative ; la reprise du traitement restaure très rapidement l'état clinique des patients. Comme le concluent les participants du DATATOP dans leur nouvelle publication, "l'étude démontre un effet bénéfique de la prise de déprényl, mais également l'absence de preuve définitive d'un effet neuroprotecteur..." ".

Le traitement de la maladie de Parkinson reste largement tributaire de la Dopa-thérapie. D'autres médicaments (agonistes dopaminergiques, anticholinergiques, etc.) peuvent être utilisés, seuls ou en association avec la L-Dopa. Le déprényl semble donc pouvoir s'adjoindre à la liste. La variabilité des résultats obtenus dans les différentes études indique cependant, pour le moins, qu'il ne s'agit pas d'un des plus efficaces. Dans l'article de $\mathrm{m} / \mathrm{s}$ de 1989, notre conclusion - optimiste indiquait l'importance que la validation du modèle expérimental "MPTP " pourrait avoir pour la compréhension des mécanismes physiopathologiques du Parkinson mais, aussi, d'autres maladies neurodégénératives. Sur ce plan, le DATATOP fait flop!

M. P.

1. Sayre LM, Arora LA, Iacofano LA, Harik SI. Comparative toxicity of MPTP, MPP + and 3,3'-dimethyl-MPDP + to dopaminergic neurons of the rat substantia nigra. Eur $J$ Pharmacol $1986 ; 124: 171-4$.

2. Fahn S. The endogenous toxin hypothesis of the etiology of Parkinson's disease and a pilot trial of high dosage antioxidants in an attempts to slow the progression of the illness. Ann NY Acad Sci 1989 ; 39 : 1109-11.

3. Allain H, Cougnard J, Neukirch HC. FSMT members. Selegiline in de novo parkinsonian patients : the French selegiline multicenter trial (FSMT). Acta Nourol Scand suppl 1991; 136 (suppl) : 73-8.

4. The Parkinson Study Group. Effects of tocopherol and deprenyl on the progression of disability in early Parkinson's disease. $N$ Engl J Med 1993 ; 328 : 176-83.

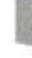

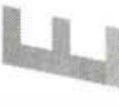

a
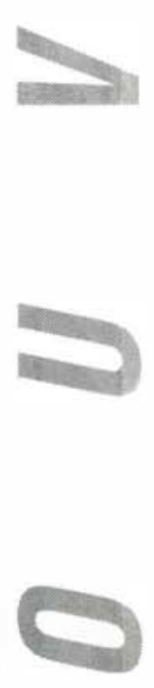